\title{
Fabrication of stretchable compliant electrodes on PDMS with Au nanoparticles
}

\author{
RITU GUPTA® \\ Department of Chemistry, Indian Institute of Technology Jodhpur, Karwar, Jodhpur 342037, India \\ ritu@iitj.ac.in
}

MS received 1 April 2018; accepted 12 June 2018; published online 16 August 2018

\begin{abstract}
A stretchable and compliant electrode surface of Au metal on polydimethylsiloxane (PDMS) is introduced in this study. A thin layer of Au nanoparticles is thus formed by a simple chemical reduction from aqueous Au salt solution with the AuPDMS gel surface itself acting as a reducing site. Employing the swelling behaviour of PDMS and Au nanoparticles affinity to bind with sulphur, an in-plane molecular device has been realized for measuring the conductance of thiol molecules. The device is capable of forming stable and robust linkages with Au. The molecules anchored between the Au islands are able to undergo reversible compression and tension, which shows the flexibility of the device.
\end{abstract}

Keywords. Au nanoparticles; PDMS; flexible; electrodes; molecular device.

\section{Introduction}

The way to future electronics is paved by the thirst for robust, flexible and stretchable soft materials for application in energy devices, diagnostics and personal healthcare [1]. Stretchable compliant electrodes have been utilized for several electrical devices, including diodes, capacitors, transistors, photodetectors, sensors, etc. [2-4]. When it comes to stretchable substrates, PDMS (polydimethylsiloxane) is the common choice of material. The compliant electrodes that make the molecules undergo stretching and compression can be an additional advantage. PDMS, being flexible, stretchable, optically transparent, electrically insulating and biocompatible, is considered ideal as a substrate for futuristic devices [2,5]. An important difference from conventional electronics is that once metallized, PDMS can integrate very well with living tissues and thus can form circuit elements in epiretinal prosthetics [6], electronic textiles, etc. [7]. PDMS can be metallized using Au over-layers; the choice of metal owes much to its biocompatibility. Metal evaporation and sputtering have been the routine methods to deposit Au film on PDMS surfaces. The Au film obtained from these methods, however, suffers from poor adhesion and spontaneous buckling, adding to undesired surface roughness. Further, such films have a tendency to develop cracks with compressive stress [8]. Metals adhere poorly to PDMS due to the low surface energy of PDMS. To address these issues, several approaches have been reported using coupling agents to enhance chemical adhesion [9], serial and selective etching to stack metal layers on PDMS [10] or manual injection and solidification of melted metals into PDMS micro-channels [11]. Even with improved adhesion, the incompatibility between PDMS and metals often causes failure in fabrication processes. Thin metallic films tend to debond from flexible PDMS substrates, and thin layers have a potential problem of irreversible cracking when bent or stressed, which is undesirable in many flexible electronics applications [12].

Several attempts have been made in the past for the fabrication of flexible metal or conductive electrodes. To avoid incompatibility issues, soft conductive materials have been utilized to embed conductive patterns in PDMS. Carbon nanotube is one of the common materials to be mixed with PDMS to form conductive composites that can be used to construct conductive and flexible micro-patterns [13]. The embedded conductive composite diffuses into the bulk PDMS polymer matrix, and therefore possible adhesion issues and cracking problems are eliminated. Several approaches have been explored to form embedded conductive PDMS patterns. Bowden et al [14] and Zhang et al [15] electrolessly prepared micro-silver electrodes inside the PDMS channels by electroless-plating-based technique, and Hao et al [16] performed region-selective electroless plating by combining the UV-induced poly(acrylic acid) grafting with electroless gold plating.

The progressive development made in the field of molecular electronics has enabled nanoscale-active elements, such as diodes, transistors, logic circuits, etc. [17]. Establishing contacts to molecules with length scale of less than a few nanometres has been one of the challenging task in molecular electronics. Molecular transport junctions are fabricated by bringing nanostructures with metal electrode pairs at a separation of a few nanometres to enable electrical contact with targeted molecules [18-21]. Several different nanofabrication methods and innovative techniques have been explored for 
creating nano-gaps to study molecular conduction. This is usually done by sophisticated techniques such as mechanical breaking, electro-migration, local oxidative cutting of carbon nanotubes, microscopic gap narrowing by electrodeposition of metal, shadow mask evaporation [22-26], etc. The usual method for making contacts with an active material is not applicable for molecular test beds, as it is prone to electrical shorts [27]. In this work, the PDMS-based compliant electrode with chemically deposited Au has been utilized for studying the molecular conduction.

\section{Experimental}

\subsection{Synthesis of AuPDMS metalized films}

AuPDMS gel was synthesized following the method developed by us previously [28]. A small portion of AuPDMS gel was dropped on the surface of aqueous $\mathrm{KAuCl}_{4}$ solution $(0.1 \mathrm{mM})$. It was allowed to spread uniformly over the liquid surface and stand for $12 \mathrm{~h}$. During this period, the bottom surface of the gel layer was in constant contact with the solution. As the gel was cured, the film was lifted gently from the solution using a support PDMS slab and further cured by heating at $180^{\circ} \mathrm{C}$.

\subsection{Characterization}

The images were recorded using a FEI Nova NanoSEM 600. Energy-dispersive spectroscopy (EDS) analysis was performed using an EDAX Genesis instrument (Mahwah, NJ) attached to the SEM column. XRD measurement on AuPDMS films was performed using a Bruker diffractometer employing $\mathrm{Cu} \mathrm{K} \alpha(\lambda=1.5406 \AA)$ radiation. The absorption measurements were carried out at room temperature using a Perkin Elmer model Lambda 900 UV-vis-NIR spectrometer and background signal from the blank PDMS was subtracted. An upright microscope with transmission and reflective mode and zooming capability of $40 \times$ was used for imaging purpose. The AuPDMS metallized film $\left(1 \mathrm{~cm}^{2}\right)$ supported on PDMS block is placed on a glass slide and Ag epoxy electrical contacts are established at both ends. Electrical measurements were performed using a Keithley 236 source measure unit.

\section{Results and discussion}

$\mathrm{Au}$ metal is electrolessly reduced on PDMS following the procedure detailed in the Experimental section and shown in figure 1. The AuPDMS gel spreads uniformly over the surface of aqueous $\mathrm{KAuCl}_{4}$ and forms a thin layer at the air-water interface as shown in figure 1 . The thin film is a multilayer film formed due to the accumulation of excess of unreacted oligomers of PDMS. The Au gets reduced chemically on PDMS surface since the surface is exposed to $\mathrm{KAuCl}_{4}$, while (a)

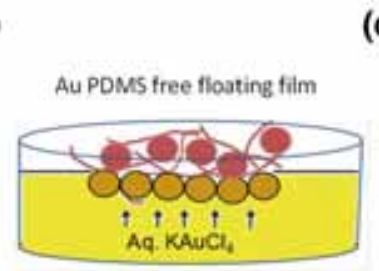

(b)
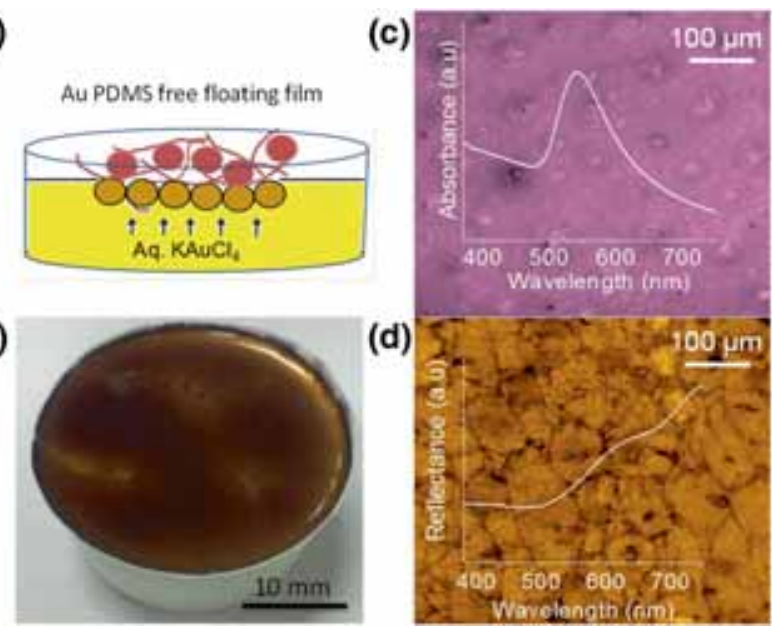

Figure 1. (a) Schematic demonstration and (b) optical photograph of the AuPDMS metalized film. Optical photographs of (c) the metalized bottom surface and (d) the top surface resembling pristine AuPDMS composite. The optical absorption spectra corresponding to top and bottom face of metalized film are laid on top in $\mathbf{c}$ and $\mathbf{d}$.
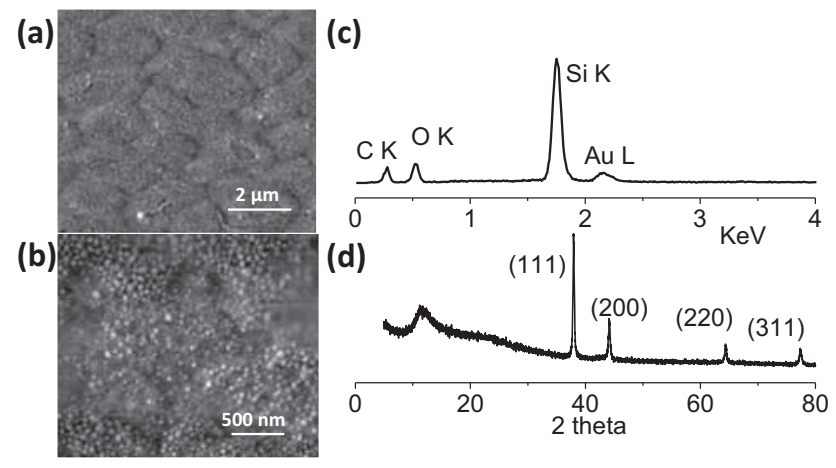

Figure 2. (a) SEM image of Au NPs reduced on PDMS surface along with (b) a magnified view. (c) EDS spectrum shows the presence of Au along with C, O and Si from PDMS. (d) The XRD pattern.

the top surface turns slightly darker (figure 1b). The metalized surface exhibits a mosaic structure with typical domain size of $\sim 2 \mu \mathrm{m}$ (figure 1c), whereas the top AuPDMS surface is non-metallized. The reflectance (inset, figure 1c) from the bottom face resembles that of a metallic Au film. However, the absorbance curve exhibits a plasmonic peak at $530 \mathrm{~nm}$ due to the presence of isolated Au nanoparticles (inset, figure 1d). Overall, the layer after this treatment is somewhat less jelly and shows a tendency to form a continuous film that cures on heating to $180^{\circ} \mathrm{C}$.

The metallized AuPDMS is further characterized. The SEM image shows the presence of dense and interconnected $\mathrm{Au}$ nanoparticles on the surface of PDMS (figure $2 \mathrm{a}$ and $\mathrm{b}$ ). Since Au nanoparticles are formed on the surface, the EDS signal corresponding to $\mathrm{Au} \mathrm{L}$ is clearly visible in the spectra in figure $2 \mathrm{c}$. The other signals $\mathrm{Si} \mathrm{K}, \mathrm{CK}$ and $\mathrm{OK}$ originate 
(a)

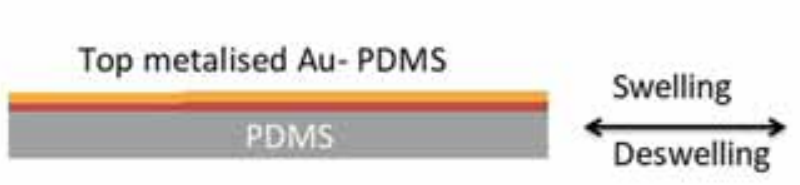

Top metalised

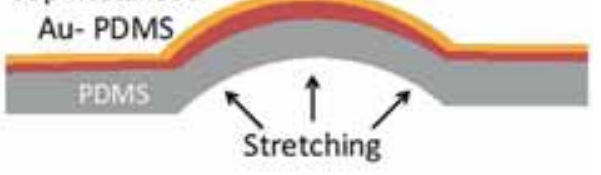

(b)
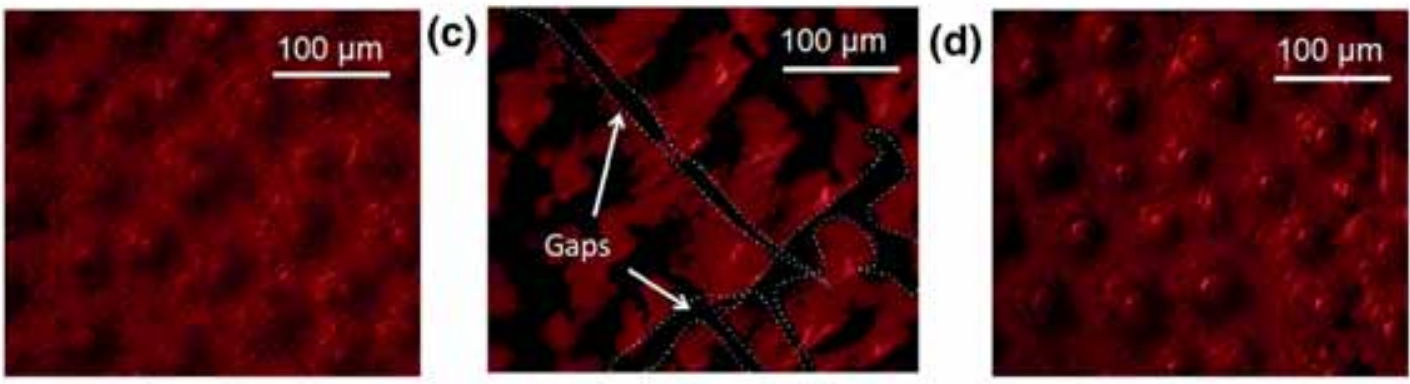

(e)
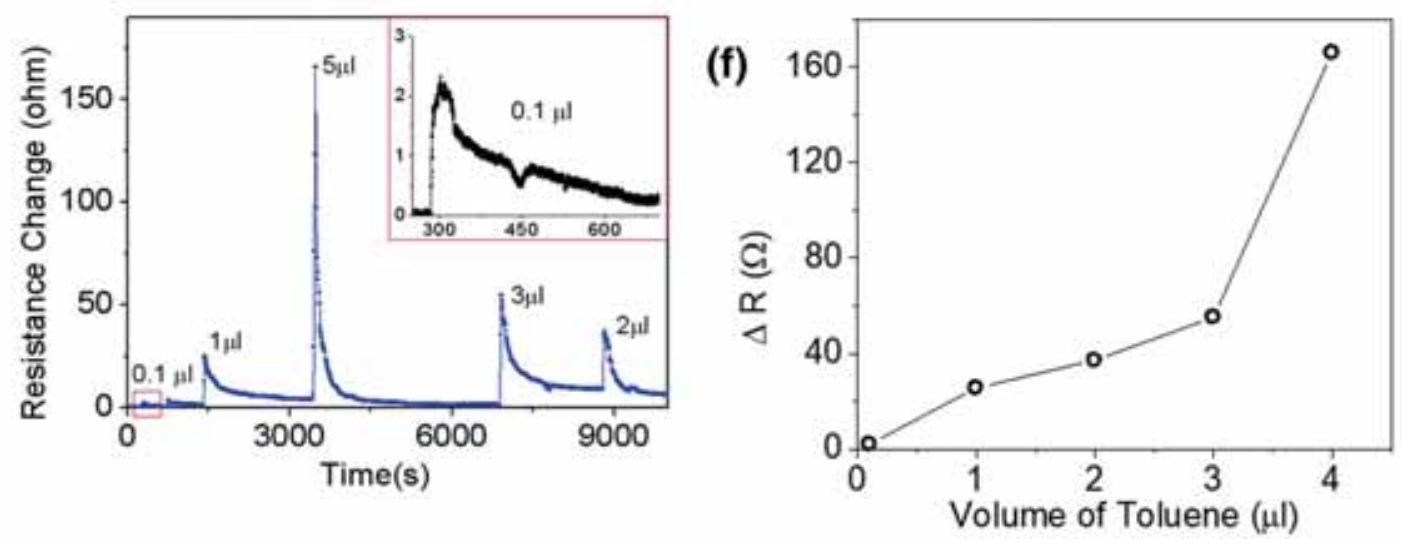

Figure 3. (a) Schematic demonstrating the reversible stretching of AuPDMS on swelling and de-swelling. (b-d) Optical microscopic images of AuPDMS film in pristine state, on swelling with toluene and after de-swelling. (e) Change in resistance with time as different amounts of toluene are injected each time. (f) Variation in resistance for different volumes of toluene used.

from PDMS matrix, in which Au nanoparticles are suspended. The XRD pattern shows intense (111) peak along with (200), (220) and (311), indicating polycrystalline Au nanoparticles in $\mathrm{Au}(0)$ state (figure $2 \mathrm{~d}$ ).

As known from our previous studies, AuPDMS undergoes buckling on swelling with toluene $[29,30]$. The buckling of PDMS exerts a tension on Au film as schematically demonstrated in figure $3 \mathrm{a}$. The optical microscopy images were collected (figure 3b-d) in situ to monitor the changes that occur on the surface of AuPDMS while it undergoes swelling. As seen in figure $3 c$, the wide gaps are clearly seen, that close reversibly on their own as toluene escapes out (figure $3 \mathrm{c}$ ). The change in resistance is studied with different volumes of toluene exposed (figure $3 \mathrm{e}$ and $\mathrm{f}$ ). The resistance increases sharply as toluene goes in the PDMS matrix, while the resistance regains slowly since de-swelling is a slower process. The jump in resistance was very steep $(\sim 165 \Omega$ per square $)$ for higher concentration $(5 \mu 1)$, while the resistance increased by $\sim 4 \Omega$ per square for $0.1 \mu 1$ of toluene.

The AuPDMS film is highly conducting with a sheet resistance of $19 \Omega$ per square, which increases to $241 \Omega$ per square on swelling with toluene. The gaps that open up on toluene swelling are utilized for trapping of thiol molecules. The thiol molecules are well known to bond strongly with Au. This property has been utilized for the assembly of thiols in between the gaps that open up in Au film on swelling of PDMS. In the swollen PDMS, thiol solution was further injected and current was monitored (figure 4e). The excessive swelling first leads to the breaking of circuit, which slowly regains once toluene evaporates. The current increases to a few $\mathrm{nA}$ and then saturates. At this point, $I-V$ measurements were performed as shown in figure $4 \mathrm{f}$. The curves show typical molecular characteristics due to the tunnelling behaviour [31]. The device is stable as seen by the several $I-V$ curves measured after every 30-min interval. The change from direct current to tunnelling current is remarkable for this type of device structure. The effect of mechanical stretching of molecules on electrical properties was further examined as shown in figure 5. For testing, the AuPDMS molecular device was exposed to a drop of toluene ( $2 \mu 1)$ to intentionally disturb the molecular junctions formed between the closed gaps by PDMS swelling. The relative 


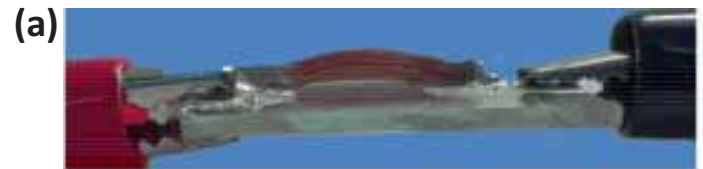

(b)

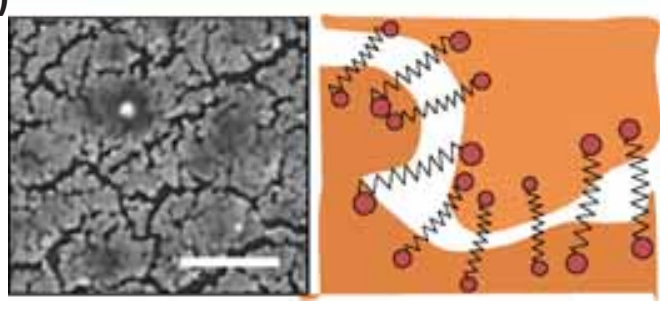

(e)

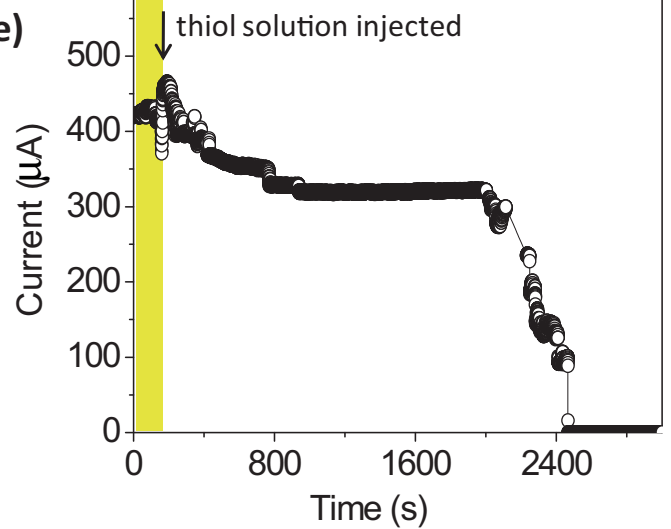

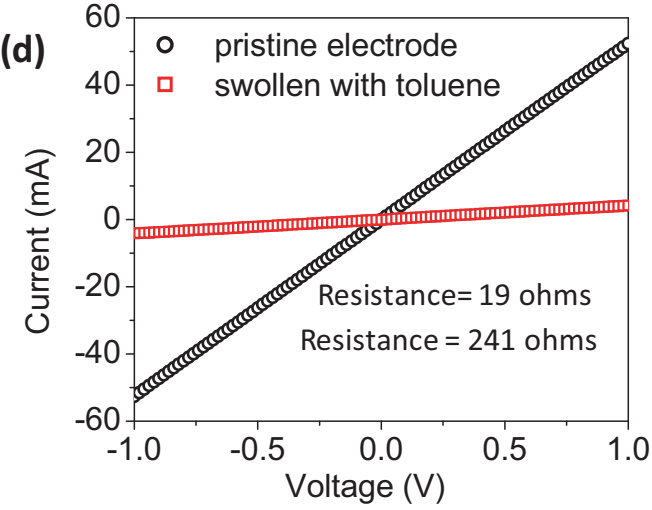

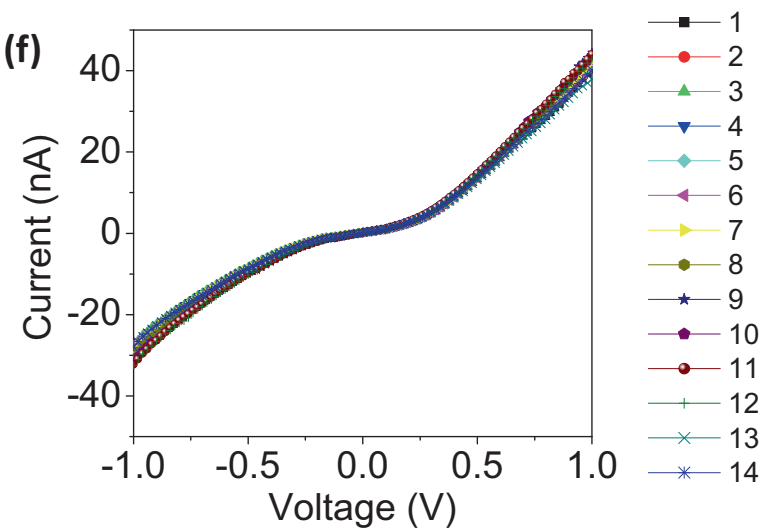

Figure 4. (a) Photograph of the molecular device. (b) SEM image of the surface where molecular junctions are formed. (c) Schematic showing thiol molecules stitched between the gaps created on swelling of AuPDMS. (d) $I-V$ characteristics of the AuPDMS before and after swelling with toluene. (e) In situ changes in current when the molecular self-assembly takes place. (f) $I-V$ characteristics of molecular device measured for several hours. The numbering refers to successive measurement after every 30-min interval.

(a)

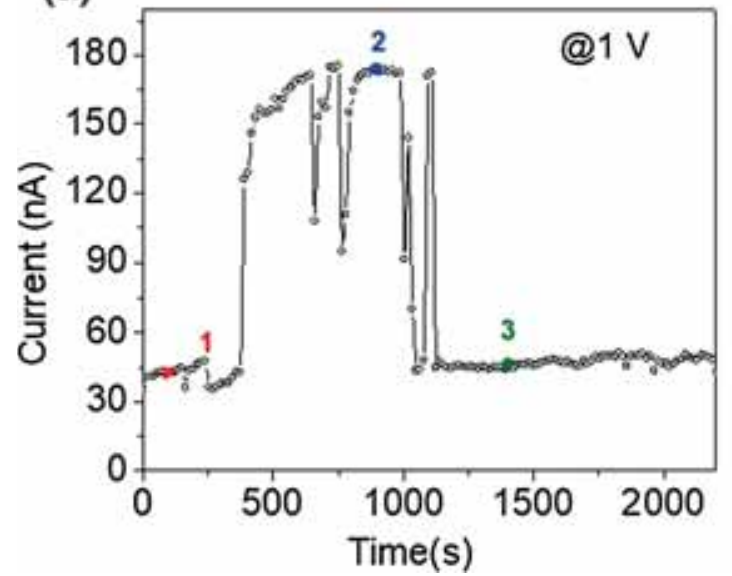

(b)

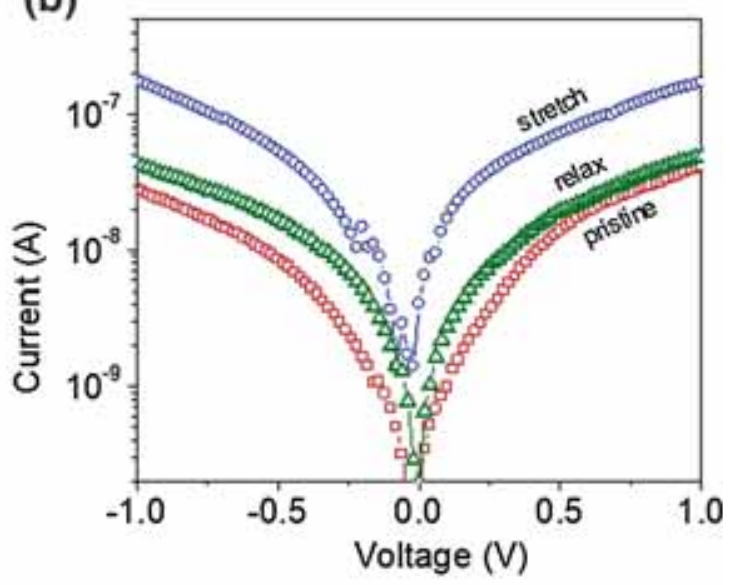

Figure 5. (a) Change in current passing through the molecules assembled in between the AuPDMS islands on exposure to toluene till the recovery of device. (b) $I-V$ characteristics of molecular device in pristine state, on exposure to toluene and after recovery at points marked as 1,2 and 3 in $\mathbf{a}$.

changes in electrical state were continuously monitored by measuring the current at an applied voltage of $1 \mathrm{~V}$. As toluene goes inside the PDMS matrix, the gaps instantly open up. As seen from figure $5 b$, the current increases due to straightening of molecular chains in the gaps that get into defects and act like scattering sites, resulting in low current. 


\section{Conclusion}

In conclusion, a molecular device could be successfully fabricated using AuPDMS as the active electrode. The molecules could be self-assembled between the Au gaps, which open up on toluene swelling. Further, one order increase in current was observed on stretching the molecular junctions. The stretching and compressing of the molecules trapped in the Au islands could be performed while measuring the current.

\section{Acknowledgements}

Ritu Gupta thanks Prof Giridhar U Kulkarni (CeNS, Bengaluru) for the technical discussions and suggestions in carrying out this work. The author also thanks Ajay B Urgunde (IIT Jodhpur) for formatting the manuscript.

\section{References}

[1] Heath J R and Ratner M A 2003 Phys. Today 5643

[2] Khang D Y, Jiang H, Huang Y and Rogers J A 2006 Science 311208

[3] Xu B, Xiao X, Yang X, Zang L and Tao N 2005 J. Am. Chem. Soc. 1272386

[4] Chen F 2005 Nano Lett. 5503

[5] McDonald J C and Whitesides G M 2002 Acc. Chem. Res. 35 491

[6] Ko H C 2008 Nature $\mathbf{4 5 4} 748$

[7] Choi M C, Kim Y and Ha C S 2008 Prog. Polym. Sci. 33581

[8] Huck W T S, Bowden N, Onck P, Pardoen T, Hutchinson J W and Whitesides G M 2000 Langmuir 163497
[9] Lee K J, Fosser K A and Nuzzo R G 2005 Adv. Funct. Mater. 15557

[10] Lim K S, Chang W J, Koo Y M and Bashir R 2006 Lab Chip 6578

[11] Siegel A C, Bruzewicz D A, Weibel D B and Whitesides G M 2007 Adv. Mater. 19727

[12] Tsuyoshi S 2010 Adv. Mater. 221831

[13] Jung Y J 2006 Nano Lett. 6413

[14] Bowden N, Brittain S, Evans A G, Hutchinson J W and Whitesides G M 1998 Nature 393146

[15] Zhang Q, Xu J, Liu Y and Chen H Y 2008 Lab Chip 8352

[16] Hao Z, Chen H and Ma D 2009 Anal. Chem. 818649

[17] Tao N J 2006 Nat. Nanotechnol. 1173

[18] Song H, Reed M A and Lee T 2011 Adv. Mater. 231583

[19] Zhou C, Deshpande M R, Reed M A, Jones I L and Tour J M 1997 Appl. Phys. Lett. 71611

[20] Joachim C and Ratner M A 2005 Proc. Natl. Acad. Sci. 102 8801

[21] Li X, He J, Hihath J, Xu B, Lindsay S M and Tao N 2006 J. Am. Chem. Soc. 1282135

[22] McCreery R L 2004 Chem. Mater. 164477

[23] Wold D J, Haag R, Rampi M A and Frisbie C D 2002 J. Phys. Chem. B 1062813

[24] Yan H, Bergren A J and McCreery R L J 2011 J. Am. Chem. Soc. 13319168

[25] Bonifas A P and McCreery R L 2010 Nat. Nanotechnol. 5 612

[26] Hsu J W P, Loo Y L, Lang D V and Rogers J 2003 J. Vac. Sci. Technol. 211928

[27] Kim T W, Wang G and Lee T 2008 IEEE Trans. Nanotechnol. 7140

[28] Gupta R and Kulkarni G U 2015 Bull. Mater. Sci. 38817

[29] Scott A, Gupta R and Kulkarni G U 2010 Macromol. Chem. Phys. 2111640

[30] Gupta R and Kulkarni G U 2011 ChemSusChem 4737

[31] Bruot C, Hihath J and Tao N 2011 Nat. Nanotechnol. 735 